\title{
A Simple Ultrasound Based Classification Algorithm Allows Differentiation of Benign from Malignant Breast Lesions by Using Only Quantitative Parameters
}

\author{
Panagiotis Kapetas, ${ }^{1}$ Ramona Woitek, ${ }^{1,2}$ Paola Clauser, ${ }^{1}$ Maria Bernathova, ${ }^{1}$ \\ Katja Pinker, ${ }^{1,3}$ Thomas H. Helbich, ${ }^{1}$ Pascal A. Baltzer ${ }^{1}$ \\ ${ }^{1}$ Department of Biomedical Imaging and Image-guided Therapy, Medical University of Vienna, Waehringer Guertel 18-20, 1090, Vienna, Austria \\ ${ }^{2}$ Department of Radiology, University of Cambridge, Cambridge Biomedical Campus, Cambridge, CB2 0QQ, UK \\ ${ }^{3}$ Molecular Imaging and Therapy Service, Memorial Sloan-Kettering Cancer Center, 301 E 55th St, New York, NY, 10022, USA
}

\begin{abstract}
Purpose: We hypothesized that different quantitative ultrasound (US) parameters may be used as complementary diagnostic criteria and aimed to develop a simple classification algorithm to distinguish benign from malignant breast lesions and aid in the decision to perform biopsy or not. Procedures: One hundred twenty-four patients, each with one biopsy-proven, sonographically evident breast lesion, were included in this prospective, IRB-approved study. Each lesion was examined with B-mode US, Color/Power Doppler US and elastography (Acoustic Radiation Force Impulse-ARFI). Different quantitative parameters were recorded for each technique, including pulsatility $(\mathrm{PI})$ and resistive Index (RI) for Doppler US and lesion maximum, intermediate, and minimum shear wave velocity $\left(S W V_{\max }, S W V_{\text {interm }}\right.$, and $\left.S W V_{\text {min }}\right)$ as well as lesion-to-fat $S W V$ ratio for ARFI. Receiver operating characteristic curve (ROC) analysis was used to evaluate the diagnostic performance of each quantitative parameter. Classification analysis was performed using the exhaustive chi-squared automatic interaction detection method. Results include the probability for malignancy for every descriptor combination in the classification algorithm.

Results: Sixty-five lesions were malignant and 59 benign. Out of all quantitative indices, maximum SWV (SWV $\max$ ), and RI were included in the classification algorithm, which showed a depth of three ramifications $\left(\mathrm{SWV}_{\max } \leq\right.$ or $>3.16$; if $S W V_{\max } \leq 3.16$ then $\mathrm{RI} \leq 0.66,0.66-0.77$ or $>0.77$; if $\mathrm{RI} \leq 0.66$ then $S W V_{\max } \leq$ or $>2.71$ ). The classification algorithm leads to an AUC of 0.887 (95\% Cl 0.818-0.937, $p<0.0001$ ), a sensitivity of $98.46 \%$ (95\% Cl $91.7-100 \%$ ), and a specificity of $61.02 \%$ (95\% Cl $47.4-73.5 \%)$. By applying the proposed algorithm, a falsepositive biopsy could have been avoided in $61 \%$ of the cases.

Conclusions: A simple classification algorithm incorporating two quantitative US parameters $\left(\mathrm{SWV}_{\max }\right.$ and RI) shows a high diagnostic performance, being able to accurately differentiate benign from malignant breast lesions and lower the number of unnecessary breast biopsies in up to $60 \%$ of all cases, avoiding any subjective interpretation bias.
\end{abstract}

Key words: Breast cancer, Ultrasound, Doppler ultrasonography, Elastography, Biopsy, Imaging biomarkers, Decision tree 


\section{Introduction}

Ultrasound (US) of the breast is an established adjunct to mammography for the detection and characterization of breast lesions. Despite its high sensitivity, breast US suffers from a low specificity, which results in a high number of false positives and a variable accuracy [1-3] by using morphologic criteria as described in the BI-RADS lexicon [4]. In addition, US is highly operator-dependent, with a generally moderate inter-reader agreement [5-7].

In order to raise the specificity of breast US, several complementary techniques have been introduced, including Doppler and elastography. Doppler evaluates tumor vascularity [3] while elastography provides information about the mechanical properties of tissue [8]. Furthermore, both techniques offer quantitative parameters (pulsed Doppler and shear wave elastography or acoustic radiation force impulse-ARFI) [9-11], which have the potential to be used as imaging biomarkers. Imaging biomarkers are parameters that can be objectively and quantitatively measured using imaging techniques, in order to detect or characterize a disease [12].

Classification algorithms aim to aid in clinical decision making by incorporating different criteria in a formalized manner [13]. Such a formalized and thus objective combination of diagnostic features in the context of a multiparametric approach is supposed to improve specificity and reduce inter-reader variability of breast US. Similar algorithms have been introduced for breast magnetic resonance imaging [14] and demonstrated successfully high diagnostic performance and improved inter-reader agreement $[15,16]$. The possibility to establish a comparable classification algorithm for breast US, by incorporating only quantitative data acquired from a multiparametric approach, has not been investigated yet. However, quantitative parameters have the potential to raise the specificity of morphological B-mode US, as well as its reproducibility $[9-11,17,18]$.

We hypothesized that different quantitative US parameters may be used as complementary diagnostic criteria and aimed to develop a simple classification algorithm to distinguish benign from malignant breast lesions and aid in the decision to perform biopsy or not.

\section{Materials and Methods}

\section{Patients}

Between October 2015 and September 2016, 124 patients (age range 18-82 years, mean age 52 years) were included in this prospective, IRB-approved, cross-sectional study. Informed consent was obtained from all individual participants included in the study. The study has been performed in accordance with the ethical standards as laid down in the 1964 Declaration of Helsinki and its later amendments. The study participants included both symptomatic and women referred to our breast center for a screening-detected abnormality. Only patients with newly diagnosed, US detected BI-RADS 4 or 5 lesions, which will undergo needle biopsy were included. Exclusion criteria were patient age less than 18 years, pregnancy or lactation, and refusal to undergo a histologic workup. In cases of more than one lesion in the same patient, only the most suspicious or the largest one was included in the study.

\section{Data Acquisition}

All examinations were performed with a Siemens Acuson S3000 device (Siemens Medical Solutions, Mountain View, CA, USA) by one out of a pool of three breast radiologists, with at least 2 years of experience in ARFI elastography and at least 3 years in breast imaging and Doppler US. Lesions were initially identified in B-mode using a linear $18 \mathrm{MHz}$ transducer (18L6HD). Lesion size was defined as the maximum lesion diameter at B-mode US.

Color and Power Doppler examinations were performed using the same transducer for the identification of vessels inside or around (at a 2-mm distance) the lesion [17]. For the Doppler examination, a region of interest (ROI) that was large enough to include the whole lesion and a small amount of surrounding tissue was used. In order to facilitate identification of small vessels, minimal compression was applied, filter settings were set as low as possible, and flow settings were also set to low [17, 19]. Color gain was slowly reduced, until background noise disappeared, in order to achieve maximum sensitivity. When vascularity was detected, pulsed Doppler was used to acquire a spectral waveform of the flow in the most prominent arterial vessel of the tumor. The Doppler angle was kept between $0^{\circ}$ and $60^{\circ}$ and no angle correction was used. Using the machine's integrated software, pulsatility (PI) and resistive index (RI) for each vessel were calculated. For this calculation, the examiner chose the best cycle from the Doppler waveform and manually placed the cursor at the maximum systolic and minimum diastolic velocities $[17,20]$.

Subsequently, the transducer was changed to a linear, $9 \mathrm{MHz}$ one (9L4) and ARFI elastography images of each lesion were acquired. For the ARFI examinations, the latest available software (namely Virtual Touch IQ-VTIQ), already installed in the device, was used. A ROI that was large enough to include the whole lesion and the surrounding tissue was drawn and minimal precompression was applied, in order to avoid artificial tissue stiffening $[8,21]$. On the acquired, color-coded image, four $2 \times 2$-mm-sized quantification ROIs were placed: one on the stiffest area of the lesion (as appreciated on the color-coded velocity map), one at an area of intermediate stiffness, and one at a soft lesion area, as well as a further one on the surrounding fatty tissue at the same depth with the lesion (if this could be included in the image), in order to measure the respective shear wave velocity (SWV) [22]. Using the available quality map, the quantification ROIs were placed on areas of high image 
quality [23], even in cases when due to a large lesion diameter (approaching the footprint of the transducer), the quality of the measurements was low in the periphery of the lesion. To avoid motion artifacts, the patients were asked in some cases to hold their breath for a couple of seconds [8]. Only one measurement was acquired for each lesion, since a large prospective study has demonstrated shear wave elastography to have an almost perfect intra-reader reproducibility [10]. The measurement scale was adjusted in order to acquire valid measurements (maximum measurable velocity $6.5-10 \mathrm{~m} / \mathrm{s}$ ) [22]. Using the acquired SWV values, a ratio of the intralesional-to-fatty tissue SWV was calculated (lesion-to-fat ratio, L/F ratio) [11].

\section{Histopathological Examination}

All patients underwent US-guided biopsy using a $14 \mathrm{G}$ biopsy system (BIP-HistoCore ${ }^{\circledR}$; BIP Medical, Tuerkenfeld, Germany). The results of the histopathological analysis of the biopsy specimen were used as the reference standard for patients with benign lesions as well as for patients with malignant lesions in case they underwent neoadjuvant chemotherapy (NAC). For patients with malignant tumors not undergoing NAC, as well as for patients receiving surgery due to a lesion with uncertain malignant potential, the post-surgical histopathology result was used as the reference standard.

\section{Statistical Analysis}

The study sample size was calculated based on a hypothetical improvement of the area under the curve (AUC) of Bmode US of the breast from 0.800 to 0.900 with a type I error of $5 \%$ and a statistical power of $80 \%$ through the addition of different quantitative parameters. The benign to malignant ratio was assumed at 0.75 , since our clinic is an assessment center for breast imaging with a consecutively high number of breast cancer patients. The values of 0.800 and 0.900 were determined as average values considering several breast US studies [3, 8, 18, 22, 24-27].

Statistical calculations were performed using the software SPSS 20 (IBM Corp, Armonk NY, USA) and MedCalc 12 (MedCalc Software bvba, Ostend, Belgium). No predefined cut-off values for the differentiation between benign and malignant lesions were used. Diagnostic accuracy of all quantitative measurements was evaluated using receiver operating characteristics (ROC) curve analysis. Classification analysis was performed using the exhaustive chisquared automatic interaction detection (CHAID) method that builds a classification tree with ramifications determined by hierarchical database splits based on chi-square test results. The final diagnostic categories or nodes are characterized by a definite probability of malignancy for specific feature combinations. Minimal parent and child node sizes were set to 10 and 5, respectively. A Bonferroni- adjusted alpha error of $5 \%$ was set as the ramification limit. The classification tree robustness was verified by 10 -fold cross-validation $[14,28]$. The cut-off values for each ramification were automatically calculated by the CHAID algorithm, based on the iterative testing of possible split values by sequential chi-squared tests. Thus, no predefined cut-off values were used for the analysis.

\section{Results}

\section{Lesion Characteristics}

Sixty-five lesions were malignant $(52.4 \%)$ and 59 were benign (47.6\%). The median size of all lesions was $13 \mathrm{~mm}$ (range 4-55 mm). The median size of the malignant lesions was $13 \mathrm{~mm}(\mathrm{Q} 19 \mathrm{~mm}, \mathrm{Q} 320 \mathrm{~mm})$ and that of the benign ones was $13 \mathrm{~mm}$ as well (Q1 $10 \mathrm{~mm}, \mathrm{Q} 322 \mathrm{~mm}$ ). There was no statistically significant difference between the median sizes between benign and malignant lesions $(p>0.05)$. Histopathological diagnoses of all lesions are summarized in Table 1.

\section{Quantitative Parameters}

The diagnostic performance of all quantitative parameters is shown in Table 2. Doppler US identified vessels in 92 out of the 124 lesions. RI was significantly higher in malignant lesions as compared to benign ones and demonstrated an area under the ROC curve (AUC) of 0.642 (cutoff $0.64, p=$ $0.016)$. The discriminatory power of PI did not reach significant levels.

All elastography quantitative parameters demonstrated significantly higher values in malignant than in benign lesions. As measured by the AUC, L/F ratio showed the highest performance (cutoff 2.06, AUC 0.873, $p<0.001$ ) followed by maximum SWV $\left(\mathrm{SWV}_{\max }\right.$ cutoff $3.20 \mathrm{~m} / \mathrm{s}$, AUC 0.867, $p>0.001$ ).

\section{Classification Algorithm}

The calculated classification algorithm is shown in Fig. 1 and its use is explained in the figure legends. The classification algorithm included two variables, namely $\mathrm{SWV}_{\max }$ and RI, and showed a depth of three ramifications. All other assessed quantitative parameters did not increase its accuracy and were not included in the algorithm.

In brief, in the first step, $\mathrm{SWV}_{\max }$ is evaluated: if it is higher than $3.16 \mathrm{~m} / \mathrm{s}$, the probability of malignancy is > $85 \%$ and biopsy is warranted. If the $\mathrm{SWV}_{\max }$ of the lesion is lower than $3.16 \mathrm{~m} / \mathrm{s}$, RI is taken into consideration: if this is higher than 0.66 , the probability of malignancy is still above $33 \%$. However, in case the RI is lower than 0.66 or no vessels can be detected in the lesion, $\mathrm{SWV}_{\max }$ is once again considered. If it is more than $2.71 \mathrm{~m} / \mathrm{s}$, the probability of malignancy surpasses $33 \%$. However, if $\mathrm{SWV}_{\max }$ is less 
Table 1. Detailed histopathological diagnoses of all lesions

\begin{tabular}{llll}
\hline Malignant lesions & $n$ & Benign lesions & $n$ \\
\hline Invasive ductal carcinoma NOS & $53(81.6 \%)$ & Fibroadenoma & $24(40.7 \%)$ \\
Invasive lobular carcinoma & $6(9.2 \%)$ & Fibroadenomatous hyperplasia & $8(13.6 \%)$ \\
Ductal carcinoma in situ & $4(6.2 \%)$ & Papilloma & $5(8.5 \%)$ \\
Mucinous carcinoma & $1(1.5 \%)$ & Fibrosis & $4(6.7 \%)$ \\
Neuroendocrine carcinoma & $1(1.5 \%)$ & Others & $18(30.5 \%)$ \\
\hline
\end{tabular}

Percentages are given in parentheses. NOS not otherwise specified

than $2.71 \mathrm{~m} / \mathrm{s}$, the probability that the lesion is malignant falls to $2.7 \%$, in which case follow-up of the lesion can be safely recommended.

The classification algorithm allowed a risk level assessment with increasing levels of malignancy (node $2>$ node $5>$ nodes 4 and $7>$ node 6 ) (Table 3 ). The classification algorithm leads to an AUC of 0.887 (95\% CI 0.818-0.937, $p<0.0001)$, a sensitivity of $98.46 \%$ (95\% CI 91.7-100\%), and a specificity of $61.02 \%$ (95\% CI $47.4-73.5 \%)$.

By applying the proposed algorithm to avoid false positives, 36 out of 59 benign lesions (61\%) could be classified correctly as benign (node 6). Subsequently, an unnecessary biopsy could have been avoided in these 36 cases. The algorithm leads to one false-negative case $(2.7 \%)$ (Grade 1 invasive ductal carcinoma) (Figs. 2 and 3).

\section{Discussion}

The results of our study demonstrate that a simple classification algorithm, taking into account two quantitative US parameters ( $\mathrm{SWV}_{\max }$ and RI,) shows a high diagnostic performance, being able to accurately distinguish benign from malignant breast lesions and substantially lower the number of unnecessary breast biopsies.

A significant shortcoming of breast US is its low specificity and positive predictive value, leading to a considerable amount of false-positive results and unnecessary biopsies [1-3]. To overcome this, US techniques other than B-mode have been developed, offering an insight into functional and molecular tissue properties. These include, among others, Doppler US and ARFI elastography, which have shown to raise the specificity of breast US $[3,11,20]$. Our cohort included overall 59 benign lesions and the proposed classification algorithm could correctly classify 36
(61 \%) as benign. This in turn means that our algorithm avoids unnecessary biopsy in 36 out of 59 cases.

Any proposed classification algorithm should provide diagnostic certainty in order to be useful in clinical practice. Indeed, our calculated algorithm could provide a definite diagnosis with a diagnostic certainty of more than $97 \%$ for almost $30 \%$ of all cases.

In addition to that, both US techniques that were used can offer quantitative parameters, which provide an objective assessment of tumor vascularity (PI and RI) and stiffness (SWV), thus limiting any subjective interpretation bias. Since the measurements are acquired by the examiner, the presence of measurement and reader bias cannot be excluded. However, several studies have shown that quantitative Doppler US and ARFI elastography are reproducible techniques, with an acceptable intra- and inter-reader agreement $[9,11,18,29]$. Still, the thresholds determined in the present exploratory study need to be confirmed and eventually adjusted to the respective center and clinical situation they are used in.

Even though it shows a high diagnostic power, one could question why such an algorithm is helpful. Its main advantage, in comparison to B-mode, is the use of quantitative parameters, which may contribute to an increased specificity and reproducibility, allowing for a high diagnostic certainty of the examiner. Obviously, this algorithm aids in the characterization of lesions, assessed at B-mode US.

A lesion classified in the terminal nodes 2, 4, 5, and 7 has a possibility of malignancy of at least $33.3 \%$. Consequently, a clinical decision to perform biopsy is appropriate. However, lesions classified in the terminal node 6 $\left(\mathrm{SWV}_{\max } \leq 2.71 \mathrm{~m} / \mathrm{s}\right.$ and $\mathrm{RI} \leq 0.66$ or no vessels detectable $)$ demonstrate a probability of malignancy of $2.7 \%$. This is only minimally higher than the cutoff for the BI-RADS 3

Table 2. Diagnostic performance of all acquired quantitative parameters

\begin{tabular}{|c|c|c|c|c|c|c|c|}
\hline Index & AUC & $p$ value & Cutoff & Sensitivity & $95 \% \mathrm{CI}$ & Specificity & $95 \% \mathrm{CI}$ \\
\hline PI & 0.540 & 0.523 & 1.90 & 77.8 & $62.9-88.8$ & 39.5 & $25.0-55.6$ \\
\hline RI & 0.642 & 0.016 & 0.64 & 78.7 & $64.3-89.3$ & 53.3 & $37.9-68.3$ \\
\hline $\mathrm{SWV}_{\max }$ & 0.867 & $<0.0001$ & 3.20 & 81.5 & $70.0-90.1$ & 88.1 & $77.1-95.1$ \\
\hline $\mathrm{SWV}_{\text {interm }}$ & 0.864 & $<0.0001$ & 2.58 & 86.4 & $75.0-94.0$ & 80.0 & $67.0-89.6$ \\
\hline $\mathrm{SWV}_{\min }$ & 0.829 & $<0.0001$ & 2.10 & 87.1 & $76.1-94.3$ & 73.2 & $59.7-84.2$ \\
\hline $\mathrm{L} / \mathrm{F}$ ratio & 0.873 & $<0.0001$ & 2.06 & 92.1 & $82.4-97.4$ & 74.1 & $61.0-84.7$ \\
\hline
\end{tabular}

$P I$ pulsatility index, $R I$ resistive index, $S W V$ shear wave velocity, $S W V_{\max }$ maximum $\mathrm{SWV}, S W V_{\text {interm }}$ intermediate $\mathrm{SWV}, S W V_{\min }$ minimum $\mathrm{SWV}$ Cut-off values of $\mathrm{SWV}_{\max }, \mathrm{SWV}_{\text {interm }}$, and $\mathrm{SWV}_{\min }$ are given in $\mathrm{m} / \mathrm{s}$. Sensitivity, specificity, and $95 \%$ confidence intervals in $\%$ 


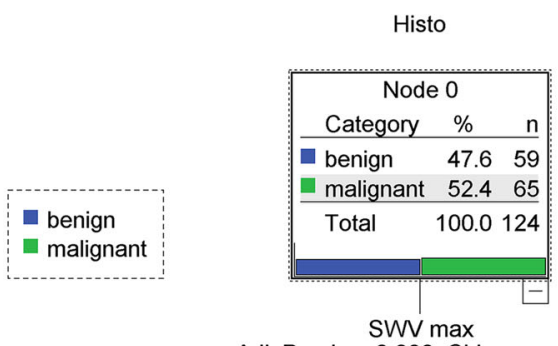

Adj. P-value $=0.000$, Chi-square $=54$.

$353, d f=1$

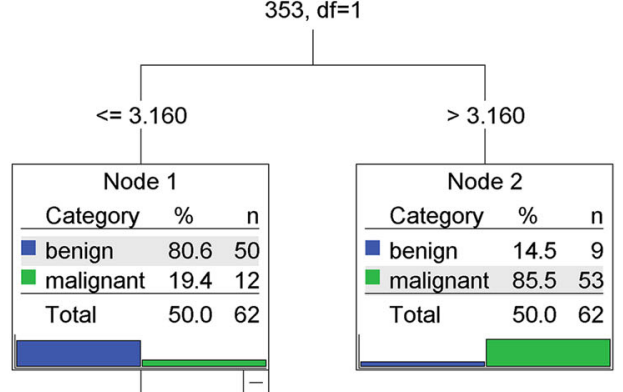

Adj. P-value $=0.011$, Chi-square $=16$

$636, \mathrm{df}=2$

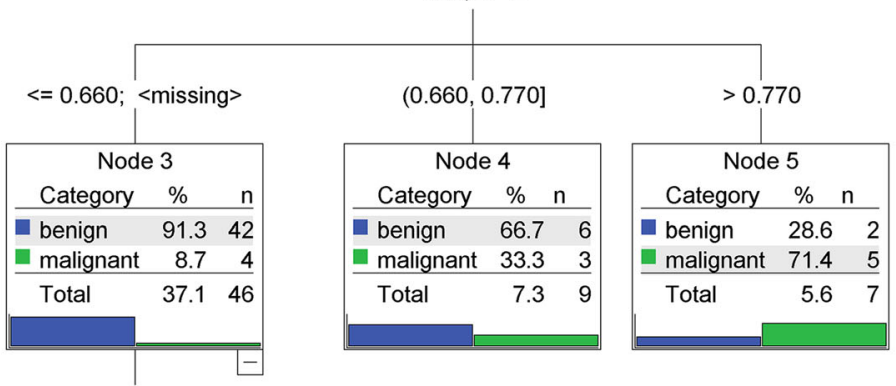

Adj. P-value $=0.034$, Chi-square $=8$.

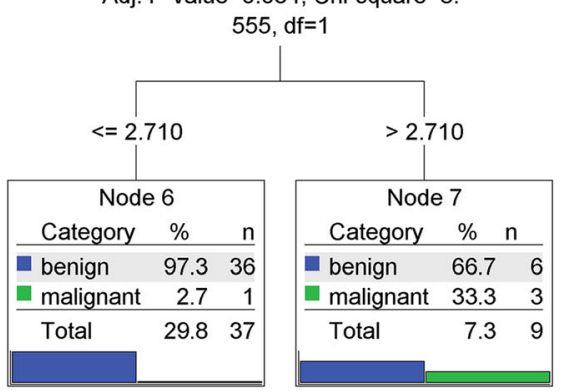

Fig. 1 The calculated classification algorithm for the differentiation of benign and malignant lesions. The study population (node 0 ) is further split into child nodes (1-7), using the variable with the highest discriminating power. After three ramifications, no further discrimination can be achieved. Blue bars demonstrate the percentage of benign and green bars that of malignant lesions in each node. In the first step, $\mathrm{SWV}_{\max }$ is taken into consideration: if $\mathrm{SWV}_{\max }$ is higher than $3.16 \mathrm{~m} / \mathrm{s}$, the probability of malignancy is $>85 \%$. If the $\mathrm{SWV}_{\max }$ is lower than $3.16 \mathrm{~m} / \mathrm{s}, \mathrm{RI}$ is taken into consideration: if the RI is higher than 0.66 , the probability of malignancy is still above $33 \%$. However, in case the RI is lower than 0.66 or no vessels can be detected, $S_{W} V_{\text {max }}$ is once again considered. If $\mathrm{SWV}_{\max }$ is higher than $2.71 \mathrm{~m} / \mathrm{s}$, the probability of malignancy surpasses $33 \%$. In contrast, if $S W V_{\text {max }}$ is less than $2.71 \mathrm{~m} / \mathrm{s}$, the probability that the lesion is benign is $97.3 \%$. $R$ I resistive index, $S W V_{\text {max }}$ maximum shear wave velocity

category $(2 \%)$ [4]. Therefore, a short-term follow-up for such lesions seems appropriate, without the risk of missing a significant number of breast cancers.

In our study, several quantitative parameters were evaluated and two of them, namely RI and $\mathrm{SWV}_{\max }$, were incorporated into the calculated classification algorithm, leading to its high AUC. Several studies have shown that malignant breast lesions demonstrate higher RI values than benign ones. This is usually attributed to the different structure of tumor vessels as compared to normal ones [20, 
Table 3. Characteristics of the nodes of the classification algorithm

\begin{tabular}{|c|c|c|c|c|c|}
\hline Node & Definition & Predicted category & $n$ malignant & $n$ benign & $n$ total \\
\hline 1 & $\mathrm{SWV}_{\max } \leq 3.16$ & benign & $12(19.4 \%)$ & $50(80.6 \%)$ & 62 \\
\hline 2 & $S W V_{\max }>3.16$ & malignant & $53(85.5 \%)$ & $9(14.5 \%)$ & 62 \\
\hline 3 & $\mathrm{SWV}_{\max } \leq 3.16$ and $\mathrm{RI} \leq 0.66$ or missing & benign & $4(8.7 \%)$ & $42(91.3 \%)$ & 46 \\
\hline 4 & $S W V_{\max } \leq 3.16$ and $R I: 0.66-0.77$ & benign & $3(33.3 \%)$ & $6(66.7 \%)$ & 9 \\
\hline 5 & $S W V_{\max } \leq 3.16$ and $R I>0.77$ & malignant & $5(71.4 \%)$ & $2(28.6 \%)$ & 7 \\
\hline 6 & $S W V_{\max } \leq 3.16$ and $R I \leq 0.66$ or missing and $S W V_{\max } \leq 2.71$ & benign & $1(2.7 \%)$ & $36(97.3 \%)$ & 37 \\
\hline 7 & $S W V_{\max } \leq 3.16$ and $R I \leq 0.66$ or missing and $S W V_{\max }>2.71$ & benign & $3(33.3 \%)$ & $6(66.7 \%)$ & 9 \\
\hline
\end{tabular}

$S W V$ shear wave velocity (in $\mathrm{m} / \mathrm{s}$ ), $R I$ resistive index

The table refers to the nodes in Fig. 1. Nodes 1 and 3 represent parent nodes, and nodes 2, 4, 5, 6 and 7 (italics) represent terminal nodes. Percentages are given in parentheses

30-32]. However, other studies demonstrated a significant overlap in RI values between benign and malignant breast lesions, making evident that RI alone has a limited role in their differentiation [17, 33, 34]. On the other hand, shear wave elastography utilizing ARFI imaging has also proven to be useful to distinguish benign from malignant breast lesions $[11,18,35]$. ARFI provides a quantitative measure of tissue stiffness, namely the velocity of shear wave-induced tissue displacement (SWV) $[23,36]$. SWV is higher in stiffer (usually malignant) tissues. According to a systematic review by Liu, et al., maximum elasticity in a $2-\mathrm{mm}$ ROI at the stiffest area of the lesion could be the most valuable parameter, which is in line to our findings [27]. Our results support the possibility of utilizing these quantitative parameters as imaging biomarkers, for the differentiation of benign from malignant breast lesions. Although L/F ratio demonstrated a slightly higher AUC than $\mathrm{SWV}_{\text {max }}$, it was not included into the algorithm by the CHAID. In our clinical experience, $\mathrm{SWV}_{\max }$ is more reliable than $\mathrm{L} / \mathrm{F}$ ratio (one measurement less) and thus probably better suited as a predictor for malignancy in clinical practice. This higher reliability of $\mathrm{SWV}_{\max }$ as compared to $\mathrm{L} / \mathrm{F}$ ratio has been demonstrated in [11].

According to a meta-analysis by Liu, et al. in 2016 [27], quantitative shear wave elastography shows a pooled sensitivity and specificity of 89 and $87 \%$ respectively. In our study, $\mathrm{SWV}_{\max }$ also demonstrated a specificity higher than the classification algorithm (88.1 vs. $61.1 \%)$. However, both sensitivity and AUC were higher when $\mathrm{SWV}_{\max }$ was combined with RI than on its own. The high specificity of $\mathrm{SWV}_{\max }$ came at a cost of a sensitivity of $81.5 \%$, thus limiting its usefulness in clinical practice. In order for a diagnostic test to be useful in clinical routine, a high sensitivity is mandatory, so as not to miss any significant number of cancers. Our aim was to establish a classification algorithm to aid clinical decision making regarding whether a biopsy should be performed or not. As the priority in trying to omit unnecessary biopsies is not to miss cancer, maintaining a high sensitivity (in our case $98.46 \%$ ) is mandatory at the cost of a somewhat lower specificity. This specificity however can be directly translated into the potential to omit unnecessary biopsies which is evidenced by the fact that the specificity of the proposed classification algorithm remains substantially higher than the usually reported specificity of B-mode breast US [3, 37].

Since in our study the elastogram was only acquired in the image with the largest lesion diameter, the possible effects of anisotropy on the measured velocities were not investigated. However, a previous study has demonstrated that anisotropy as such is not plane related [38]. In this study, the number of both benign and malignant lesions demonstrating higher SWVs in the radial plane was almost equal to the ones with higher SWVs in the antiradial plane. Since our images were
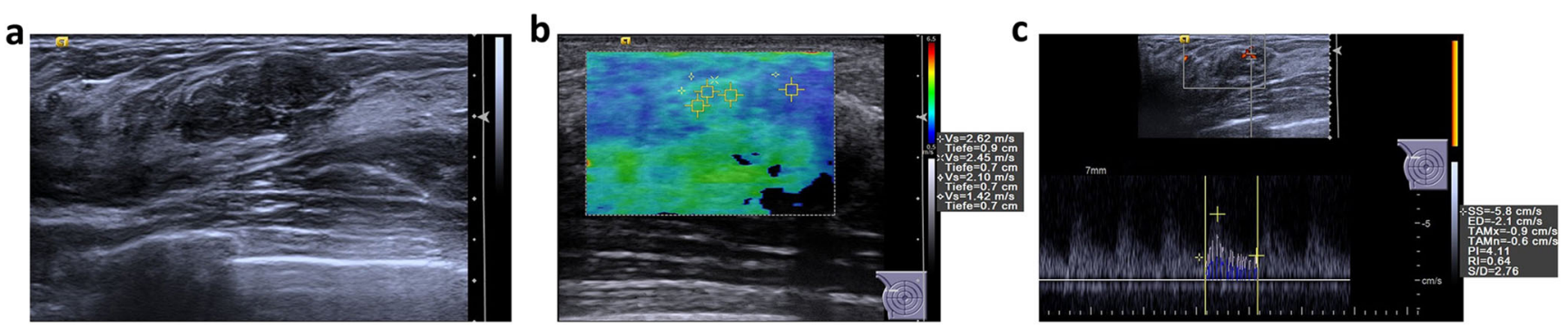

Fig. 2 Multiparametric ultrasound of a palpable, 20-mm large lesion in the craniolateral right breast of a 23-year-old patient. a In B-mode, the lesion was heterogeneously hypoechoic, lobulated, with a partially indistinct margin and was classified as BIRADS 4a. b In VTIQ elastography, the lesion was overall quite soft, with a SWVmax of $2.62 \mathrm{~m} / \mathrm{s}$. c Power Doppler detected internal vascularity and the spectrum analysis of the most prominent artery demonstrated an Rl of 0.64 . The application of the classification algorithm would classify this lesion as node 6 , with probability of malignancy of $2.7 \%$, making a follow-up instead of biopsy a safe alternative. Ultrasound guided biopsy revealed an area of sclerosing adenosis 

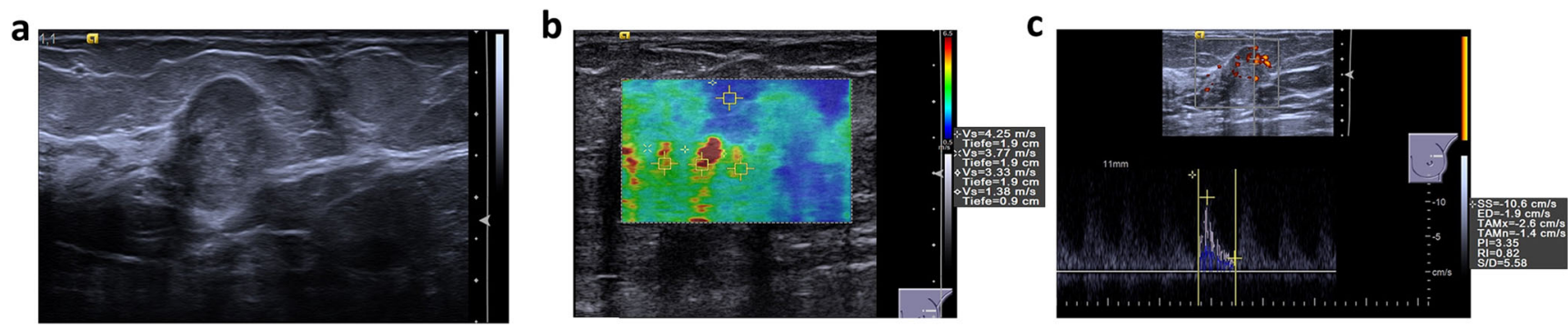

Fig. 3 Multiparametric ultrasound of a palpable, 17-mm large lesion in the craniolateral left breast of a 70-year-old patient. a In B-mode, the lesion was isoechoic, slightly heterogeneous, with a partially indistinct margin and an anti-parallel orientation and was classified as BI-RADS 4c. b In VTIQ elastography, the lesion demonstrated hard parts, with a SWVmax of $4.25 \mathrm{~m} / \mathrm{s}$. c Power Doppler showed abundant internal vascularity and the spectrum analysis of the most prominent artery demonstrated an $\mathrm{RI}$ of 0.82 . The application of the classification algorithm would classify this lesion as node 2 , with a very high probability of malignancy. Ultrasound guided biopsy revealed a Grade 1 mucinous carcinoma

acquired irrespective of the radial or antiradial plane and the included lesions grew in all different planes, it may be assumed that the effects of anisotropy were averaged, when taking into consideration the whole patient cohort.

Our study had some limitations. First, our study population included a high percentage of malignant lesions $(52.4 \%$ of all patients), possibly leading to some degree of spectrum bias. The reason is that it stemmed from an assessment center, where a higher than in the average population pretest probability of malignancy is to be expected. Moreover, we chose to include only cases with a histopathological verification. This aimed at providing a robust standard of reference; however, it may also introduce a sampling bias, since cases with a more straightforward benign diagnosis were excluded from our analysis. Finally, this is a monocentric study with a relatively low number of patients. Although the examinations were performed by different radiologists, trained in different hospitals and with varying levels of experience, our data still need to be tested in a larger validation study, with more participants and examiners. It would be of highest clinical relevance to apply our classification algorithm in a larger number of BI-RADS 3 and 4 lesions to validate its potential to reduce the number of unnecessary benign breast biopsies.

\section{Conclusions}

In conclusion, a simple classification algorithm incorporating two quantitative US parameters ( $\mathrm{SWV}_{\max }$ and RI) shows a high diagnostic performance, being able to accurately differentiate benign from malignant breast lesions and lower the number of unnecessary breast biopsies in up to $60 \%$ of all cases, avoiding any subjective interpretation bias.

Acknowledgements. Open access funding provided by Medical University of Vienna.

Compliance with Ethical Standards

\section{Conflict of Interest}

The authors declare that they have no conflict of interest.
Open Access This article is distributed under the terms of the Creative Commons Attribution 4.0 International License (http://creativecommons.org/licenses/by/4.0/), which permits unrestricted use, distribution, and reproduction in any medium, provided you give appropriate credit to the original author(s) and the source, provide a link to the Creative Commons license, and indicate if changes were made.

\section{References}

1. Nothacker M, Duda V, Hahn M, Warm M, Degenhardt F, Madjar H, Weinbrenner S, Albert US (2009) Early detection of breast cancer: benefits and risks of supplemental breast ultrasound in asymptomatic women with mammographically dense breast tissue. A systematic review. BMC Cancer 9:335

2. Madjar H (2010) Role of breast ultrasound for the detection and differentiation of breast lesions. Breast Care 5:109-114

3. Cho N, Jang M, Lyou CY, Park JS, Choi HY, Moon WK (2012) Distinguishing benign from malignant masses at breast US: combined US elastography and color doppler US-influence on radiologist accuracy. Radiology 262:80-90

4. Mendelson EB, Böhm-Vélez M, Berg WA, et al. (2013) ACR BIRADS ${ }^{\circledR}$ Ultrasound. In ACR BI-RADS $®$ Atlas, Breast Imaging Reporting and Data System, Eds D'Orsi CJ, Sickles EA, Mendelson EB, Morris EA Reston, VA: American College of Radiology, pp $216-355$

5. Scaperrotta G, Ferranti C, Costa C, Mariani L, Marchesini M, Suman L, Folini C, Bergonzi S (2008) Role of sonoelastography in nonpalpable breast lesions. Eur Radiol 18:2381-2389

6. Calas MJ, Almeida RM, Gutfilen B, Pereira WC (2010) Intraobserver interpretation of breast ultrasonography following the BI-RADS classification. Eur J Radiol 74:525-528

7. Park CS, Kim SH, Jung NY, Choi JJ, Kang BJ, Jung HS (2015) Interobserver variability of ultrasound elastography and the ultrasound BI-RADS lexicon of breast lesions. Breast Cancer 22:153-160

8. Berg WA, Cosgrove DO, Dore CJ et al (2012) Shear-wave elastography improves the specificity of breast US: the BE1 multinational study of 939 masses. Radiology 262:435-449

9. Kumar A, Srivastava V, Singh S, Shukla RC (2010) Color Doppler ultrasonography for treatment response prediction and evaluation in breast cancer. Future Oncol 6:1265-1278

10. Cosgrove DO, Berg WA, Dore CJ et al (2012) Shear wave elastography for breast masses is highly reproducible. Eur Radiol 22:1023-1032

11. Kapetas P, Pinker-Domenig K, Woitek R, Clauser P, Bernathova M, Spick C, Helbich T, Baltzer PA (2017) Clinical application of acoustic radiation force impulse imaging with virtual touch IQ in breast ultrasound: diagnostic performance and reproducibility of a new technique. Acta Radiol 58:140-147

12. Bickel H, Pinker-Domenig K, Bogner W, Spick C, Bagó-Horváth Z, Weber M, Helbich T, Baltzer P (2015) Quantitative apparent diffusion coefficient as a noninvasive imaging biomarker for the differentiation 
of invasive breast cancer and ductal carcinoma in situ. Investig Radiol 50:95-100

13. Harper PR (2005) A review and comparison of classification algorithms for medical decision making. Health Policy 71:315-331

14. Baltzer PA, Dietzel M, Kaiser WA (2013) A simple and robust classification tree for differentiation between benign and malignant lesions in MR-mammography. Eur Radiol 23:2051-2060

15. Marino MA, Clauser P, Woitek R, Wengert GJ, Kapetas P, Bernathova M, Pinker-Domenig K, Helbich TH, Preidler K, Baltzer PAT (2016) A simple scoring system for breast MRI interpretation: does it compensate for reader experience? Eur Radiol 26:2529-2537

16. Woitek R, Spick C, Schernthaner M, Rudas M, Kapetas P, Bernathova M, Furtner J, Pinker K, Helbich TH, Baltzer PAT (2017) A simple classification system (the Tree flowchart) for breast MRI can reduce the number of unnecessary biopsies in MRI-only lesions. Eur Radiol 27:3799-3809

17. Ozdemir A, Ozdemir H, Maral I et al (2001) Differential diagnosis of solid breast lesions: contribution of Doppler studies to mammography and gray scale imaging. J Ultrasound Med 20:1091-1101 quiz 1102

18. Golatta M, Schweitzer-Martin M, Harcos A et al (2014) Evaluation of virtual touch tissue imaging quantification, a new shear wave velocity imaging method, for breast lesion assessment by ultrasound. Biomed Res Int 2014:960262

19. Raza S, Baum JK (1997) Solid breast lesions: evaluation with power Doppler US. Radiology 203:164-168

20. del Cura JL, Elizagaray E, Zabala R, Legórburu A, Grande D (2005) The use of unenhanced Doppler sonography in the evaluation of solid breast lesions. AJR Am J Roentgenol 184:1788-1794

21. Wojcinski S, Brandhorst K, Sadigh G, Hillemanns P, Degenhardt F (2013) Acoustic radiation force impulse imaging with virtual touch tissue quantification: measurements of normal breast tissue and dependence on the degree of pre-compression. Ultrasound Med Biol 39:2226-2232

22. Li DD, Xu HX, Guo LH, Bo XW, Li XL, Wu R, Xu JM, Zhang YF, Zhang K (2016) Combination of two-dimensional shear wave elastography with ultrasound breast imaging reporting and data system in the diagnosis of breast lesions: a new method to increase the diagnostic performance. Eur Radiol 26:3290-3300

23. Tozaki M, Saito M, Benson J, Fan L, Isobe S (2013) Shear wave velocity measurements for differential diagnosis of solid breast masses: a comparison between virtual touch quantification and virtual touch IQ. Ultrasound Med Biol 39:2233-2245

24. Gokalp G, Topal U, Kizilkaya E (2009) Power Doppler sonography: anything to add to BI-RADS US in solid breast masses? Eur J Radiol 70:77-85

25. Berg WA, Zhang Z, Lehrer D et al (2012) Detection of breast cancer with addition of annual screening ultrasound or a single screening
MRI to mammography in women with elevated breast cancer risk. JAMA 307:1394-1404

26. Athanasiou A, Tardivon A, Tanter M et al (2010) Breast lesions: quantitative elastography with supersonic shear imaging - preliminary results. Radiology 256:297-303

27. Liu B, Zheng Y, Huang G, Lin M, Shan Q, Lu Y, Tian W, Xie X (2016) Breast lesions: quantitative diagnosis using ultrasound shear wave elastography - a systematic review and meta-analysis. Ultrasound Med Biol 42:835-847

28. Kass GV (1980) An exploratory technique for investigating large quantities of categorical data. Appl Stat 29:119-127

29. Kim SY, Woo S, Hwang SI, Moon MH, Sung CK, Lee HJ, Cho JY, Kim SH (2014) Usefulness of resistive index on spectral Doppler ultrasonography in the detection of renal cell carcinoma in patients with end-stage renal disease. Ultrasonography 33:136-142

30. Stanzani D, Chala LF, Barros N, Cerri GG, Chammas MC (2014) Can Doppler or contrast-enhanced ultrasound analysis add diagnostically important information about the nature of breast lesions? Clinics (Sao Paulo) 69:87-92

31. Gupta K, Chandra T, Kumaresan M, et al. (2017) Role of Color Doppler for assessment of malignancy in solid breast masses: a prospective study. International Journal of Anatomy, Radiology and Surgery 6:RO59-RO65

32. Kettenbach J, Helbich TH, Huber S, Zuna I, Dock W (2005) Computer-assisted quantitative assessment of power Doppler US: effects of microbubble contrast agent in the differentiation of breast tumors. Eur J Radiol 53:238-244

33. Hooley RJ, Scoutt LM, Philpotts LE (2013) Breast ultrasonography: state of the art. Radiology 268:642-659

34. Youssefzadeh S, Eibenberger K, Helbich T, Jakesz R, Wolf G (1996) Use of resistance index for the diagnosis of breast tumours. Clin Radiol 51:418-420

35. Ianculescu V, Ciolovan LM, Dunant A, Vielh P, Mazouni C, Delaloge S, Dromain C, Blidaru A, Balleyguier C (2014) Added value of Virtual Touch IQ shear wave elastography in the ultrasound assessment of breast lesions. Eur J Radiol 83:773-777

36. Balleyguier C, Canale S, Ben Hassen W, Vielh P, Bayou EH, Mathieu MC, Uzan C, Bourgier C, Dromain C (2013) Breast elasticity: principles, technique, results: an update and overview of commercially available software. Eur J Radiol 82:427-434

37. Zengin B, Elverici E, Barca N, Cavusoglu M, Duran S, Ozsoy A, Aktas H (2013) Positive predictive values of the sonographic BIRADS final assessment categories for breast lesions. J Breast Health 9:125-129

38. Skerl K, Vinnicombe S, Thomson K, McLean D, Giannotti E, Evans A (2016) Anisotropy of solid breast lesions in 2D shear wave elastography is an indicator of malignancy. Acad Radiol 23:53-61 